\title{
Características de personalidade e depressão em idosas da Universidade para a Terceira Idade (UNITI/UFRGS)
}

\author{
Characteristics of personality and depression in elderly women of the University \\ for the Third Age
}

\author{
Tatiana Quarti Irigaray ${ }^{1}$, Rodolfo Herberto Schneider ${ }^{2}$ \\ ${ }^{1}$ Psicóloga. Mestre e Doutoranda em Gerontologia Biomédica, Pontifícia Universidade Católica do Rio Grande do Sul (PUCRS), Porto Alegre, RS. \\ 2 Médico geriatra. Doutor em Medicina, PUCRS. Professor adjunto, Instituto de Geriatria e Gerontologia, PUCRS. \\ Este trabalho foi realizado no Instituto de Geriatria e Gerontologia, PUCRS.
}

\section{Resumo}

Introdução: Os aspectos de personalidade têm sido apontados como fatores que podem contribuir para o desenvolvimento de depressão na velhice. O objetivo deste estudo foi examinar a influência da personalidade no surgimento de sintomas depressivos em idosas.

Método: O estudo teve um delineamento transversal, com a participação de 103 idosas, com idade entre 60 e 86 anos e escolaridade mínima de sete anos de estudo, integrantes da Universidade para a Terceira Idade (UNITI/UFRGS), e o processo de amostragem foi o de conveniência. Todas as participantes preencheram o Termo de Consentimento Livre e Esclarecido e responderam a respeito de informações sociodemográficas, fatores de personalidade (Inventário Fatorial de Personalidade IFP) e depressão (Escala de Depressão Geriátrica - GDS).

Resultados: Observou-se a presença de dois grupos (clusters) com características distintas de personalidade: um mais preocupado com os outros (cluster 1) e outro mais voltado para si (cluster 2). Embora ambos os grupos não tenham preenchido os critérios para depressão, ocorreu uma diferença significativa no escore total da GDS, em que o grupo mais preocupado com os outros (cluster 1) apresentou maior intensidade de sintomatologia depressiva.

Conclusões: O estudo aponta para uma associação entre dimensões de personalidade e depressão em idosas. As idosas agrupadas no cluster 1, que são mais voltadas para o outro, mais deferentes, organizadas, persistentes e interativas, apresentaram uma maior intensidade de sintomatologia depressiva em relação àquelas do cluster 2, que são mais voltadas para si próprias, menos interativas e pouco dominantes. Outros estudos são sugeridos.

Descritores: Personalidade, depressão, idosas, velhice.

\begin{abstract}
Introduction: Personality aspects have been mentioned as factors that may contribute for the development of depression in old age. This study aimed at examining the influence of personality in the onset of depressive symptoms in elderly women.

Method: The study had a cross-sectional design and included 103 elderly women, aged between 60-86 years and with minimum schooling level of 7 years, attending the University for the Third Age (UNITI/UFRGS), using a convenience sample. All the participants signed a consent form and provided answers about sociodemographic information, personality factors (Factor Personality Inventory - FPI) and depression (Geriatric Depression Scale - GDS).
\end{abstract}


Results: Presence of two groups (clusters) with distinct personality characteristics was observed: one more preoccupied with the others (cluster 1) and the other more self-concerned (cluster 2). Although both groups did not meet the criteria for depression, there was a significant difference in total GDS score, in which the group more preoccupied with the others (cluster 1) presented more depressive symptoms.

Conclusions: The study indicates an association between personality dimensions and depression in elderly women. The elderly women in cluster 1, who were more devoted to the others, more deferential, organized, persistent and interactive, presented more depressive symptoms in relation to those in cluster 2 , who were more self-centered, less interactive and less dominant. Others studies are suggested.

Keywords: Personality, depression, elderly women, old age.

\section{Introdução}

As dimensões de personalidade podem contribuir para a adaptação ao envelhecimento, influenciando a saúde e a longevidade na velhice ${ }^{1}$. Embora as bases dessa associação ainda sejam pouco conhecidas, há evidências da importância da personalidade no bemestar subjetivo e no início de depressão em idosos. O termo personalidade não apresenta uma definição única, variando de acordo com a teoria de personalidade empregada. De acordo com a teoria de Murray $^{2}$, a personalidade refere-se a uma série de eventos que abrangem toda a vida do indivíduo, refletindo tanto elementos duradouros e recorrentes do comportamento quanto elementos novos e únicos. Para ele, a personalidade atuaria como o agente organizador ou governador do indivíduo ${ }^{2}$.

Segundo $\mathrm{Neri}^{3}$, a personalidade tem relação com as maneiras como os indivíduos geralmente se comportam, têm experiências, acreditam e se sentem em relação a si mesmos, aos outros e ao mundo. Kaplan et al. ${ }^{4}$ definem a personalidade como a totalidade de traços emocionais e comportamentais que caracterizam o indivíduo nas situações de vida do dia-a-dia, sob condições normais, sendo relativamente estável e previsível. Para Hilgard et al. ${ }^{5}$, a personalidade é composta por características individuais de comportamento que são responsáveis pelo ajustamento do indivíduo ao ambiente.

Inúmeras teorias tentam explicar o desenvolvimento da personalidade na velhice. As primeiras pesquisas mostraram que a personalidade se tornava mais rígida com o envelhecimento e que se desenvolvia muito pouco na velhice ${ }^{6,7}$. Estudos posteriores, surgidos a partir da década de 70 , sugeriram que ocorreria uma estabilidade dos traços de personalidade ao longo da vida adulta e da velhice ${ }^{8-}$ ${ }^{11}$. Desta forma, a personalidade não se tornaria mais rígida, mas permaneceria da mesma forma que sempre fora desde a vida adulta.

Alguns estudos tendem a considerar que diferenças entre coortes se refletem na personalidade de idosos ${ }^{12,13}$. Pessoas de coortes mais recentes parecem ser mais flexíveis, ou seja, apresentam menor rigidez, do que as de coortes anteriores ${ }^{12}$. Estes achados sugerem que aumentos de rigidez verificados em estudos anteriores podem estar relacionados à influência cultural que uma geração sofreu durante a idade adulta e, provavelmente, as futuras gerações de idosos serão capazes de se adaptar mais facilmente ao processo de envelhecimento do que as gerações anteriores ${ }^{14}$.

Já teorias atuais de personalidade entendem o desenvolvimento humano como um processo multidimensional e multidirecional, sendo composto pela ocorrência conjunta de ganhos, perdas e estabilidades. De acordo com este modelo, as pessoas possuem potenciais para a mudança (plasticidade) e para a manutenção e recuperação da adaptação perante eventos limitadores e facilitadores do processo de desenvolvimento normal durante toda a vida ${ }^{15-21}$. Assim, na velhice, os traços de personalidade seriam modificáveis, principalmente porque nessa fase de vida as pessoas têm maior probabilidade de serem confrontadas com eventos de vida estressantes que requerem adaptação. Embora muitos indivíduos possam manter a personalidade estável sob circunstâncias de vida normais, infere-se que, diante de situações mais complexas, a personalidade mudaria para se adaptar às transformações da vida ${ }^{17}$.

$\mathrm{Na}$ velhice, um transtorno de humor freqüente é a depressão, apresentando uma prevalência de 4,8 a $14,6 \%$ em idosos que vivem na comunidade e uma prevalência mais alta entre idosos hospitalizados ou institucionalizados, atingindo até $22,0 \%$ desta população. Estudos que avaliam sintomas clinicamente relevantes de depressão nesse grupo etário através das escalas de sintomas, que não preenchem critérios suficientes do CID-10 ou do DSM-IV, demonstram uma prevalência na comunidade que varia de 6,4 a $59,3 \%{ }^{22}$. Embora a depressão seja um problema de saúde freqüente entre idosos ${ }^{23}$, ainda é pouco diagnosticada e tratada.

Segundo estudos ${ }^{24-27}$, a personalidade pode atuar como um dos principais fatores desencadeantes de sintomatologia depressiva na velhice. Baixos níveis de dominância e altos de neuroticismo têm sido relacionados com desencadeamento de depressão em idosos ${ }^{27}$. Por outro lado, altos níveis de extroversão e 
baixo níveis de neuroticismo aparecem associados com reduzido risco de mortalidade na velhice ${ }^{28}$. Outro estudo evidenciou que indivíduos que são otimistas e esperançosos tendem a apresentar melhor saúde, a viver mais, a ter mais humor positivo e mais sucesso do que indivíduos pessimistas, que demonstram uma maior probabilidade de desenvolver depressão e apresentar pior estado de saúde ${ }^{29}$.

O presente estudo teve por objetivo examinar a influência de características de personalidade no desencadeamento de sintomatologia depressiva em idosas.

\section{Método}

\section{Participantes}

A amostra foi composta por 103 mulheres com idade a partir dos 60 anos e escolaridade mínima de sete anos de estudo. As participantes foram recrutadas na Universidade para a Terceira Idade (UNITI/ UFRGS), através do método de conveniência. Todas as idosas integrantes da UNITI $(\mathrm{n}=153)$ foram convidadas a participar do estudo, mas apenas 109 consentiram em fazê-lo. Foram excluídas seis idosas, por apresentarem idade inferior a 60 anos.

A Universidade para a Terceira Idade (UNITI/ UFRGS) é um Projeto de Extensão, Ensino e Pesquisa do Instituto de Psicologia da Universidade Federal do Rio Grande do Sul. Seus participantes são pessoas da comunidade com sessenta anos ou mais, e sua dinâmica de trabalho aborda questões cognitivas, sociais e comunitárias, levando os idosos a pensar e repensar o seu papel enquanto cidadãos. Na UNITI, os idosos são incentivados a conquistar, manter e preservar a autonomia, uma boa qualidade de vida e a independência. Também são estimulados a adquirir novas informações e ampliar conhecimentos.

\section{Instrumentos}

Os instrumentos utilizados foram os seguintes:

\section{Ficha de dados sociodemográficos}

A ficha de dados sociodemográficos incluiu as seguintes variáveis: idade, estado civil, escolaridade, renda, ocupação anterior, ocupação atual, percepção de saúde, realização de atividade física e tempo de participação na UNITI.

\section{Avaliação de fatores de personalidade}

Inventário Fatorial de Personalidade - IFP ${ }^{30}$. É um inventário de personalidade objetivo, que avalia 15 necessidades ou motivos psicológicos (assistência, dominância, ordem, denegação, intracepção, desempenho, exibição, heterossexualidade, afago, mudança, persistência, agressão, deferência, autonomia e afiliação). O IFP é composto por 155 itens, que constituem afirmações às quais o indivíduo deve responder, utilizando alternativas de uma escala do tipo Likert, que varia progressivamente de 1 (nada característico) até 7 (totalmente característico). Foi utilizada a versão do instrumento em português, aprovada pelo Conselho Federal de Psicologia, adaptada e validada para uso no Brasil pela equipe do Prof. Luiz Pasquali ${ }^{30}$.

\section{Intensidade de sintomas depressivos}

Escala de Depressão Geriátrica - GDS-15 $5^{31}$. A GDS é uma das medidas mais utilizadas para o rastreamento de depressão em idosos. Foi utilizada a versão curta do instrumento em português, composta por 15 perguntas com respostas classificadas em sim ou não. $\mathrm{O}$ escore total da GDS é constituído a partir do somatório das respostas assinaladas pelos examinandos nos 15 itens, sendo zero o menor escore possível e 15 o maior. A versão utilizada foi adaptada de Yesavage et al. ${ }^{31}$, sendo considerada uma medida válida e confiável para uso em amostra brasileira.

\section{Procedimentos}

\section{Procedimentos para coleta dos dados}

Inicialmente, todas as idosas preencheram o Termo de Consentimento Livre e Esclarecido e, a partir daí, foram convidadas a responder acerca de informações sociodemográficas, fatores de personalidade ${ }^{30} \mathrm{e}$ sintomatologia de depressão $0^{31}$.

Os dados sociodemográficos, o IFP e a GDS foram obtidos por meio de auto-administração. Contudo, um auxiliar de pesquisa (estudante de Psicologia) sempre esteve à disposição das idosas para qualquer esclarecimento. As aplicações dos instrumentos foram feitas em grupos de até 15 participantes.

O projeto de pesquisa foi devidamente examinado e aprovado pelo Comitê de Ética em Pesquisa da Pontifícia Universidade Católica do Rio Grande do Sul, Porto Alegre, RS, Brasil ( ${ }^{\circ}$ 06/0309).

\section{Procedimentos para análise dos dados}

A descrição das variáveis foi realizada por meio das freqüências absolutas e relativas, bem como média e desvio padrão.

As associações entre os fatores de personalidade do IFP e a variável depressão (GDS) foram medidas pelo coeficiente de Correlação de Spearman. 
O teste $t$ de Student foi utilizado para a comparação realizada entre a variável depressão (GDS) e os clusters do IFP.

Foram consideradas significativas as associações com valores de $\mathrm{p} \leq 0,05$.

Para análise dos dados, foi utilizado o programa SPSS para ambiente Windows, versão 13.0.

\section{Resultados}

A amostra foi composta por 103 mulheres idosas na faixa etária entre 60-86 anos, idade média de 69,2 (desvio padrão, $\mathrm{DP}=6,46$ ). $\mathrm{O}$ grupo mais freqüente foi o de viúvas $(44,7 \%)$. Em termos de escolaridade, prevaleceu o ensino superior $(40,8 \%)$ e a renda mais presente foi de 6 a 10 salários mínimos $(38,8 \%)$. A profissão de professora foi a ocupação anterior mais freqüente $(28,1 \%)$ e a aposentadoria, a ocupação atual prevalente $(73,8 \%)$. A percepção de saúde predominante foi a de saudável $(81,6 \%)$, a maioria das idosas realiza atividade física $(74,8 \%)$ e participa do grupo de terceira idade há mais de um ano $(84,4 \%)$.

No IFP, o grupo de idosas investigado apresentou, de modo geral, características medianas para todos os fatores de personalidade, tendo como dimensões mais referidas a alta deferência (média $=50,68 ; \mathrm{DP}=6,42$ ) e baixa agressão (média $=24,58 ; \mathrm{DP}=7,53$ ). Nos outros fatores de personalidade, o grupo obteve os seguintes resultados: assistência (média $=51,59 ; \mathrm{DP}=7,04$ ), intracepção (média $=44,69 ; \mathrm{DP}=7,78)$, afago (média $=$ 45,$84 ; \mathrm{DP}=8,74$ ), afiliação (média $=55,06 ; \mathrm{DP}=6,64$ ), dominância (média $=30,31 ; \mathrm{DP}=8,64$ ), denegação (média $=37,98 ; \mathrm{DP}=6,94$ ), desempenho (média $=47,17$; $\mathrm{DP}=7,49)$, exibição (média $=28,61 ; \mathrm{DP}=8,46)$, ordem (média $=50,17 ; \mathrm{DP}=8,07$ ), persistência (média $=45,90$; $\mathrm{DP}=8,74)$, mudança (média $=47,74 ; \mathrm{DP}=7,41) \mathrm{e}$ autonomia (média $=45,04 ; \mathrm{DP}=7,08$ ). Salienta-se que o fator heterossexualidade não foi avaliado, por não apresentar relevância quanto aos objetivos deste estudo.

Na Escala de Depressão Geriátrica, observouse ausência de sintomatologia depressiva (média = 2,$65 ; \mathrm{DP}=2,42$ ), já que a amostra avaliada não apresentou escores suficientes para pontuar depressão, o que corresponderia a, no mínimo, 5 pontos. As idosas apresentaram uma pontuação mínima de 0 e máxima de 9 na GDS.

A Tabela 1 mostra as correlações encontradas entre os fatores do IFP e a variável depressão (GDS). Por meio do Coeficiente de Correlação de Spearman, obtiveram-se correlações significativas, apesar de fracas, entre os fatores afago e agressão do IFP e a variável depressão (GDS), e uma correlação negativa significativa, embora fraca, entre o fator persistência do IFP e a variável depressão (GDS).
Tabela 1 - Correlações significativas encontradas entre os fatores do Inventário Fatorial de Personalidade e a variável depressão (Escala de Depressão Geriátrica) (n $=103$ )

\begin{tabular}{lc}
\hline Fatores & Escala de Depressão Geriátrica* \\
\hline Afago & $0,212^{\dagger}$ \\
Agressão & $0,268^{\ddagger}$ \\
Persistência & $-0,212^{\dagger}$ \\
\hline${ }^{*}$ Coeficiente de correlação de Spearman. \\
${ }^{\dagger} \mathrm{p} \leq 0,05 ;{ }^{\dagger} \mathrm{p} \leq 0,01$.
\end{tabular}

Na Tabela 2, é possível observar uma análise de clusters dos fatores de personalidade do IFP. Os clusters foram usados para explorar mais as características de personalidade, buscando maximizar as diferenças entre elas no grupo estudado. Conforme pode ser observado, o cluster 1 é formado por pessoas mais voltadas para o outro, mais deferentes, organizadas, persistentes e interativas. De forma diferente, o cluster 2 é composto por pessoas mais voltadas para si próprias, menos interativas e pouco dominantes. Na mesma Tabela 2, também são apresentados os resultados da comparação entre a variável depressão (GDS) e os clusters do IFP. Por meio do teste $t$ de Student, foi possível verificar uma diferença significativa $(\mathrm{p} \leq 0,01)$ para a variável depressão (GDS) nos clusters 1 e 2. Conforme essa comparação, as pessoas agrupadas no cluster 1 (mais voltadas para o outro, mais deferentes, organizadas, persistentes e interativas) apresentaram mais sintomas de depressão em relação àquelas do cluster 2 (mais voltadas para si próprias, menos interativas e pouco dominantes).

\section{Discussão}

Conforme a apresentação anterior dos resultados, foi feita uma análise de clusters para explorar mais as diferenças entre aspectos de personalidade no grupo estudado. A partir de uma comparação entre os clusters dos fatores de personalidade (IFP) e a variável depressão (GDS), observou-se que as idosas agrupadas no cluster 2, que são mais voltadas para si próprias, menos interativas e pouco dominantes, apresentaram menos sintomas de depressão em relação àquelas do cluster 1, que são mais voltadas e interessadas no outro, mais deferentes, organizadas, persistentes e interativas. Embora os grupos que compõem o cluster 1 e o cluster 2 tenham diferido quanto à intensidade de sintomatologia depressiva, este resultado não apresenta um impacto clinicamente relevante, pois os dois grupos apresentaram ausência de sintomatologia depressiva, 
Tabela 2 - Descrição (média) dos clusters do Inventário Fatorial de Personalidade e comparação entre a variável depressão (Escala de Depressão Geriátrica) e os clusters do IFP

\begin{tabular}{lccc}
\hline Fatores IFP & Cluster 1 (n= 61) & Cluster 2 (n= 42) & p \\
\hline Assistência & 53,93 & 48,19 & $<0,001$ \\
Intracepção & 48,28 & 39,48 & $<0,001$ \\
Afago & 49,70 & 40,24 & $<0,001$ \\
Deferência & 53,28 & 46,90 & $<0,001$ \\
Afiliação & 57,67 & 51,26 & $<0,001$ \\
Dominância & 33,80 & 25,24 & $<0,001$ \\
Denegação & 39,51 & 35,76 & 0,007 \\
Desempenho & 49,98 & 43,07 & $<0,001$ \\
Exibição & 32,49 & 22,98 & $<0,001$ \\
Agressão & 27,64 & 20,14 & $<0,001$ \\
Ordem & 53,59 & 45,21 & $<0,001$ \\
Persistência & 49,46 & 40,74 & $<0,001$ \\
Mudança & 50,16 & 44,24 & $<0,001$ \\
Autonomia & 47,20 & 41,93 & $<0,001$ \\
Escala de Depressão Geriátrica & $3,11(2,76)$ & $1,98(1,64)$ & $0,01 *$ \\
\hline
\end{tabular}

O cluster 1 é formado por pessoas mais voltadas para o outro, mais deferentes, organizadas, persistentes e interativas. O cluster 2 é composto por pessoas mais voltadas para si próprias, menos interativas e pouco dominantes.

$* \mathrm{p} \leq 0,01$.

o que corresponde a uma média inferior a 5 pontos na GDS.

De acordo com os resultados do presente estudo, o cluster 1 é formado por indivíduos com grandes desejos e sentimentos de piedade, que gostam de respeitar, admirar e obedecer um superior, que tendem a pôr todas as coisas em ordem com precisão, que buscam terminar um trabalho iniciado e que gostam de dar e receber afeto de amigos. Estas características de personalidade foram as variáveis mais consistentemente associadas com uma maior intensidade de sintomatologia depressiva entre as idosas desta amostra. A associação entre aspectos de personalidade e depressão em idosos tem sido encontrada em diversos estudos $^{24-27,32}$. Um estudo ${ }^{27}$ evidenciou que baixa dominância e altos níveis de neuroticismo aparecem relacionados com início de depressão em idosos. Assim, a personalidade atuaria como um fator desencadeante de depressão na velhice, mais do que o estado de saúde física e os recursos sociais disponíveis ${ }^{27}$.

Uma das hipóteses propostas a respeito da referida associação é a de que, por terem características de personalidade mais voltadas para o outro, estas idosas apresentam expectativas irrealísticas em relação às outras pessoas, esperando receber mais afeto e apoio emocional do que realmente recebem. Assim, como não encontram a correspondência afetiva esperada, acabam desenvolvendo um maior número de sintomas depressivos. Estudos de Neri corroboram esta idéia demonstrando que a percepção subjetiva da qualidade do relacionamento é fundamental para a satisfação com a vida, as relações sociais e a saúde mental e física na velhice $^{3}$.

Uma segunda hipótese é a de que as características de personalidade das idosas do cluster 1 poderiam exercer efeitos diretos sobre o senso de auto-eficácia. Deste modo, estas dimensões de personalidade determinariam uma diminuição do senso de autoeficácia, o que levaria a uma maior expressão de sintomas depressivos. $\mathrm{Neri}^{3}$ defende, da mesma maneira, que quanto melhor o senso de auto-eficácia mais satisfação e menos depressão na velhice. Assim, quanto mais estruturado o sistema de crenças sobre as possibilidades de controlar a si próprio e ao ambiente, maior o ajustamento do idoso ${ }^{3}$.

Estudos têm mostrado a influência do papel das crenças de auto-eficácia sobre vários domínios do funcionamento, tais como aprendizagem, trabalho, esporte, saúde, ajustamento social e bem-estar ${ }^{33}$. A autoeficácia refere-se à crença que o indivíduo tem em suas capacidades de executar determinadas tarefas, controlar eventos do mundo e alcançar determinados resultados, sendo que estas crenças resultam em realização e motivação ${ }^{34}$.

Alguns autores tendem a considerar que uma velhice bem-sucedida depende, em grande parte, das 
crenças dos idosos nas capacidades para administrar a própria vida e os eventos do mundo externo ${ }^{3,33}$. Para $\mathrm{Neri}^{3}$, o senso de auto-eficácia e um estilo de vida ativo contribuem para um bom desempenho físico e mental na velhice. Por outro lado, a perda de força e a mudança de foco das crenças de auto-eficácia aumentam a vulnerabilidade ao estresse e à depressão do idoso, dificultando o seu acesso ao suporte social que necessita ${ }^{33}$.

Outro achado deste estudo é o de que as idosas avaliadas apresentaram aspectos medianos para todos os fatores de personalidade, tendo como dimensões mais referidas a alta deferência e baixa agressão. A alta deferência caracteriza pessoas que demonstram respeito, admiração e reverência a um indivíduo que julgam superior, gostando de elogiá-lo, admirá-lo, bem como de imitá-lo e obedecê-lo. Já a baixa agressão define os indivíduos que não são caracterizados pela raiva, irritação e ódio, que não gostam de superar com vigor a oposição e nem possuem o desejo de lutar, brigar, atacar, fazer oposição, censurar e ridicularizar os outros.

A explicação para este achado poderia estar no fato de que as idosas dessa amostra fazem parte de uma coorte que foi, essencialmente, educada para desempenhar os papéis de esposa, mãe e dona-de-casa. Esse resultado concorda com os dados da pesquisa sobre a história das mulheres no Brasil ${ }^{35}$, que evidenciou que, na família-modelo dos anos de 1950, os homens tinham autoridade e poder sobre as mulheres e eram os responsáveis pelo sustento da esposa e dos filhos. O casamento pressupunha uma hierarquia na qual o marido era o chefe, detentor de poder sobre a esposa e os filhos. A mulher ideal era definida a partir dos papéis femininos tradicionais de dona-de-casa, mãe e esposa. $\mathrm{O}$ instinto materno, a pureza, a resignação e a doçura faziam parte da essência feminina, sem possibilidades de contestação. A vocação para a maternidade e a vida doméstica eram marcas da feminilidade, enquanto a participação no mercado de trabalho, a força e o espírito de aventura definiam a masculinidade. A menina era educada para ser boa mãe e dona de casa exemplar ${ }^{35}$. Da mesma forma, um outro estudo ${ }^{36}$ defende a idéia de que a educação não prepara as pessoas para o envelhecimento, produzindo indivíduos com baixos recursos para o desenvolvimento tardio. As pessoas chegam à velhice com um conjunto fragilizado de disposições, devido à ênfase excessiva em identificações sociais, com o trabalho e com a procriação, o que acaba reduzindo o potencial humano do desenvolvimento ${ }^{36}$.

No presente estudo, as idosas avaliadas apresentaram ausência de depressão. Uma possível explicação para este achado talvez seja o fato de que a nossa amostra foi composta por idosas que participam de um grupo onde são estimuladas a manter atividades cognitivas e sociais, o que pode resultar em um maior sentimento de bem-estar e atuar como um possível fator protetor contra a depressão na velhice. Essa evidência está de acordo com a teoria da atividade, que diz que, quanto mais ativos os idosos se mantêm, melhor eles envelhecem ${ }^{14}$. De acordo com esta teoria, os papéis adultos são fontes importantes de satisfação, quanto maior a perda de papéis devido à aposentadoria, à viuvez, à saída dos filhos de casa, às doenças, menos satisfeito será o indivíduo. As pessoas que estão envelhecendo bem mantêm o máximo possível de atividades e encontram substitutos para os papéis perdidos $^{37}$. Uma outra hipótese de explicação para o resultado de ausência de depressão seria a de que, quando buscam a UNITI, as idosas já chegam com poucos sintomas depressivos ou ausência deles, o que as levaria a uma grande motivação para se engajarem em novas atividades. Ou ainda, que a ausência de sintomas depressivos seria mais influenciada pelo tempo de participação no grupo do que pelo estado de humor com que as idosas chegam na UNITI.

Entre as possíveis limitações deste estudo, cabe ressaltar que o mesmo foi conduzido com uma amostra formada somente por mulheres idosas, oriundas de um mesmo contexto, bem como o fato da amostra ter sido realizada por conveniência, o que pode não refletir a realidade dos idosos da própria comunidade.

\section{Conclusões}

As características de personalidade podem tanto contribuir para a manutenção da saúde e o bem-estar subjetivo na velhice quanto influenciar o desencadeamento de sintomas depressivos em idosos. Segundo resultados desse estudo, as idosas com características de personalidade mais voltadas para si próprias, menos interativas e pouco dominantes, apresentaram menos sintomas de depressão em relação àquelas que são mais voltadas e interessadas no outro, mais deferentes, organizadas, persistentes e interativas.

Observamos também que as idosas avaliadas apresentaram características medianas para todos os fatores de personalidade, tendo como dimensões mais referidas a alta deferência e baixa agressão. Em geral, a personalidade das idosas desta amostra foi caracterizada pelo respeito, admiração e reverência a um indivíduo que consideram como superior, gostando de elogiá-lo e honrá-lo, bem como imitá-lo e obedecê-lo. Por outro lado, a agressão não se mostrou como uma característica marcante desse grupo de idosas, pois elas não foram caracterizadas pela raiva, irritação e ódio e nem pelo desejo de superar com vigor a oposição. 
As participantes desse estudo não apresentaram como característica a presença de depressão. Provavelmente, a busca pela UNITI ocorra por pessoas que não estão deprimidas ou talvez o grupo exerça um papel importante na conquista e manutenção de um envelhecimento bem sucedido, bem como no desencadeamento de sintomatologia depressiva.

Outros estudos são sugeridos a fim de confirmar ou levantar outras hipóteses acerca de quais variáveis de personalidade podem estar relacionadas à depressão em idosos. Além disso, há escassez na literatura sobre a associação entre aspectos de personalidade e depressão em idosos.

\section{Referências}

1. Baltes PB, Lindenberger U, Staudinger UM. Life-span theory in developmental psychology. In: Damon W, ed. Handbook of child psychology. Vol. 1. Lerner RM, ed. Theoretical models of human development. New York: Wiley; 1998. p. 1029-143.

2. Hall CS, Lindzey G, Campbell JB. Teorias da personalidade. Porto Alegre: Artmed; 2000

3. Neri AL. Palavras-chave em gerontologia. 2. ed. Campinas: Alínea; 2005 .

4. Kaplan HI, Sadock BJ, Grebb JA. Compêndio de psiquiatria: ciências do comportamento e psiquiatria clínica. 7. ed. Porto Alegre: Artmed; 1997.

5. Hilgard ER, Atkinson RL, Atkinson RC. Instroduction to psychology. 7. ed. New York: Harcout Brace Jovanovich; 1979.

6. Papalia DE, Olds SW. Desenvolvimento humano. Porto Alegre: Artmed; 2000

7. Tavares A. Compêndio de neuropsiquiatria geriátrica. Rio de Janeiro: Guanabara Koogan; 2005.

8. McCrae RR, Costa PT Jr. Validation of five-factor model of personality across instruments and observers. J Pers Soc Psychol. 1987;52(1):81-90.

9. McCrae RR, Costa PT Jr. The stability of personality: observations and evaluations. Curr Dir Psychol Sci. 1994;3(6):173-5.

10. Herbst JH, McCrae RR, Costa PT Jr., Feaganes JR, Siegler IC. Selfperceptions of stability and change in personality at midlife: the UNC alumni heart study. Assessment. 2000;7(4):379-88.

11. Costa PT Jr., Herbst JH, McCrae RR, Siegler IC. Personality at midlife: stability, intrinsic maturation and response to life events. Assessment. 2000;7(4):365-78.

12. Schaie KW, Willis SL. Adult personality and psychomotor performance: cross-sectional and longitudinal analysis. J Gerontol. 1991;46(6):P275-84.

13. Ruth JE, Coleman PG. Personality and aging: coping and management of the self in later life. In: Birren JE, Schaie KW, eds. Handbook of the psychology of aging. 4th ed. San Diego: Academic; 1996. p. 308-22.

14. Papalia DE, Olds SW, Feldman RD. Desenvolvimento humano. Porto Alegre: Artmed; 2006.

15. Freire AS. Envelhecimento bem-sucedido e bem-estar psicológico. In: Neri AL, Freire AS, orgs. E por falar em boa velhice. Campinas: Papirus; 2000. p. 21-31.
16. McAdams DP. What do we know when we know a person? J Pers. 1995;63(3):365-96

17. Maiden RJ, Peterson SA, Caya M, Hayslip B Jr. Personality changes in the old-old: a longitudinal study. J Adult Dev. 2003;10(1):31-9.

18. Field D, Millsap RE. Personality in advanced old age: continuity or change? J Gerontol. 1991;46(6):P299-308.

19. Labouvie-Vief G, Diehl M, Tarnowski A, Shen J. Age differences in adult personality: findings from the United States and China. J Gerontol B Psychol Sci Soc Sci. 2000;55(1):P4-17.

20. Ravenna H, Jones C, Kwan VS. Personality change over 40 years of adulthood: hierarchical linear modeling analyses of two longitudinal samples. J Pers Soc Psychol. 2002;83(3):752-66.

21. Schaie KW, Willis SL. Adult development and aging. 4th ed. New York: Harpers \& Collins; 1996.

22. Frank MH, Rodrigues NL. Depressão, ansiedade, outros distúrbios afetivos e suicídio. In: Freitas EV, Py L, Cançado FAX, Doll, J, Gorzoni, ML, orgs. Tratado de geriatria e gerontologia. 2. ed. Rio de Janeiro: Guanabara Koogan; 2006. p. 376-86.

23. Argimon IIL, Stein LM. Habilidades cognitivas em indivíduos muito idosos: um estudo longitudinal. Cad Saude Publica. 2005;21(1):64-72.

24. Martin P, Long MV, Poon LW. Age change and differences in personality traits and states of the old and very old. J Gerontol B Psychol Sci Soc Sci. 2002;57(2):P144-52.

25. Small BJ, Hertzog C, Hultsch DF, Dixon RA; Victoria Longitudinal Study. Stability and change in adult personality over 6 years: findings from the Victoria Longitudinal Study. J Gerontol B Psychol Sci Soc Sci. 2003;58(3):P166-76.

26. Watson D, Walker LM. The long-term stability and predictive validity of trait measures of affect. J Pers Soc Psychol. 1996;70(3):567-77.

27. Steunenberg B, Beekman AT, Deeg DJ, Kerkhof AJ. Personality and the onset of depression in late life. J Affect Disord. 2006;92(23):243-51.

28. Wilson RS, Krueger KR, Gu L, Bienias JL, Mendes de Leon CF, Evans DA. Neuroticism, extraversion, and mortality in a defined population of older persons. Psychosom Med. 2005;67(6):841-5.

29. Hoyer WJ, Roodin PA. Adult development and aging. 5th ed. New York: McGraw-Hill; 2003.

30. Pasquali L, Azevedo MM, Ghesti I. Inventário fatorial de personalidade: manual técnico e de avaliação. São Paulo: Casa do Psicólogo; 1997.

31. Yesavage JA, Brink TL, Rose TL, Lum O, Huang V, Adey M, et al. Development and validation depression screening scale: a preliminary report. J Psychiatr Res. 1982-1983;17(1):37-49.

32. Diener E, Diener C. Most people are happy. Psychol Sci. 1996;7(3):181-5.

33. Bandura A. Self-efficacy: the exercise of control. New York: WH Freeman; 1997.

34. Rabelo DF. Auto-eficácia. In: Neri AL. Palavras-chave em gerontologia. Campinas, SP: Alínea; 2005. p. 19-23.

35. Bassanezi C. Mulheres dos anos dourados. In: Del Priore M, ed História das mulheres no Brasil. São Paulo: Contexto; 1997. p. 60739.

36. Both A. Longevidade e educação: fundamentos e práticas. In: Freitas EV, Py L, Cançado FAX, Doll J, Gorzoni ML, eds. Tratado de geriatria e gerontologia. 2. ed. Rio de Janeiro: Guanabara Koogan; 2006. p. 1446-56.

37. Neugarten BL, Havighurst R, Tobin S. Personality and patterns of aging. In: Neugarten BL, ed. Middle age and aging. Chicago: University of Chicago; 1968. p. 173-7. 